\title{
Double "Fistula-Patch" Therapy for a Patient with Intestinal and Colonic Enterocutaneous Fistulas
}

\author{
Gefei Wang, Jianan Ren*, Zhiwu Hong, Xiuwen Wu, Huajian Ren, Jun Chen, Ranran Li and Jieshou Li \\ Department of Surgery, Jinling Hospital, Medical School of Nanjing University, Nanjing, China
}

Keywords: Entero atmospheric fistula; Acute abdominal obstructions; Gastrointestinal

\section{Introduction}

Entero Cutaneous Fistula (ECF) is abnormal connections between the gastrointestinal tract and the skin, which associated with considerable morbidity and mortality. Recent case series suggest a mortality rate of 6 to $33 \%$, with sepsis and complicated malnutrition being the most common causes of death [1]. Despite advances in antimicrobial chemotherapy, nutritional support, and peri-operative critical care, the development of an ECF continues to be a major therapeutic challenge. Specific problems are the control of sepsis, maintenance of adequate fluid and electrolyte balance, provision of adequate and complication-free nutritional support, and skin-stoma care [2].

Nutrition therapy especially enteral nutrition plays a central role in the effective management of ECF [3]. In current literature, several methods have been established generally focusing on wound care and nutrition therapy especially like fistuloclysis, which is a safe, reliable, and inexpensive means of providing adequate nutrition support $[4,5]$, but it still needs special medical care.

We had reported our work, which named as "fistula-patch" technique for controlling contamination of intestinal fistula draining into the open abdominal wounds, and applying enteral nutrition. It is a simple method to control Entero Atmospheric Fistula (EAF), with the benefits of avoiding loss of enteric fistula effluent, simplifying of wound management, ceasing of tissue destruction, and affording enteral nutrition application [6]. The "fistula-patch" technique can also be a method to control ECF, and herein we report a case of double "fistulapatch" therapy for a patient with intestinal and colonic ECF.

\section{Case Report}

A 39-year-old woman presented with worsening generalized abdominal pain, distention, and emesis was admitted to local hospital. Four months ago she just had a left ovarian resection because of borderline serous cystadenoma. The patient was taken to the operating room and underwent exploratory laparotomy after eight days of conservative treatment for acute abdominal obstructions. Serious intra-abdominal extensive adhesions and distal small intestine adhered to pelvic cavity were found during laparotomy, which may result to intestinal obstructions. Resection of adhesive small intestine and repair of damaged transverse colon were performed. On the $3^{\text {rd }}$ postoperative (PO) day, disruption of abdominal incision and effusion of intestinal juice (output $>0.8 \mathrm{~L} /$ day) under incision were found.

Then the patient suffered severe electrolyte imbalances, malnutrition, and local infection. On the $26^{\text {th }}$ PO day, she was transferred to our hospital. An intestinal ECF and a colonic ECF was found under abdominal incision, which then were confirmed by fistulogram (Figure 1). The intestinal and colonic ECFs were treated with our "fistula-patch" technique, respectively. The flexible and soft silica gel lamellar (Zheyi Company Ltd., Jiangsu, China) with $0.1 \mathrm{~mm}$ in its thickness was shaped in accordance to the size of ECF and the internal form of intestinal canal nearby the fistula. Because of the elasticity of the silica gel, it could be unfolded along the long axis of intestinal tube. It was embedded through the fistulae site into the intestinal tube, acting like a patch inside the fistula (fistula-patch). Then this patch could against the inner layer of the intestine, sealing off the fistula and restoring gastrointestinal continuity (Figure 2). The sutures here were to fix the patch with the rail (drainage tube in Figure 2D) that crossed the ECF, avoiding any migrations of the patch.

There was no effusion of succus entericus or feces out from stoma after "fistula-patch" therapy. The effect of "fistula-patch" therapy for the intestinal and colonic ECF was confirmed by barium enema examination and CT examination (Figure 3). Enteral nutrition was supplied through nasogastric tube and wound care was simplified. Definitive operation for two ECFs was administrated, after 3 months of enteral nutrition, the patient recovered without any postoperatively complications.

\section{Discussion}

The management of ECF patients can be divided into three phases. The first phase is to control sepsis, and to maintain the balance of adequate fluid and electrolytes. The second phase includes the development of an interim plan for wound care and nutrition therapy.

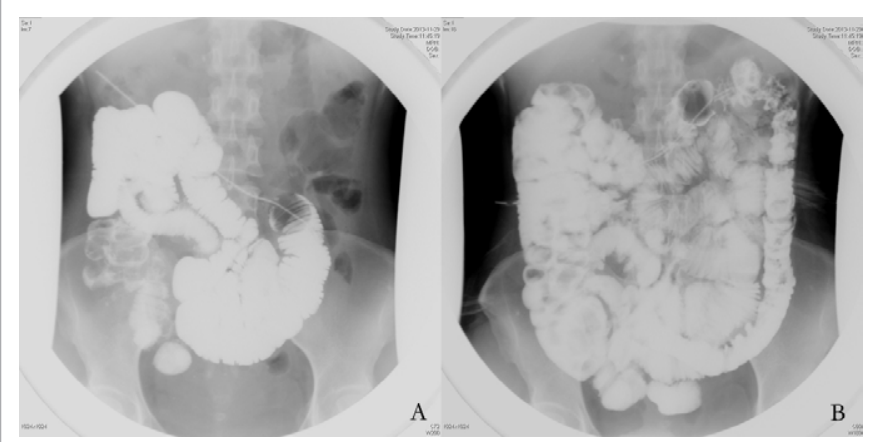

Figure 1: The ECFs were confirmed by fistulogram. (A) The intestinal ECF; (B) The colonic ECF.

*Corresponding author: Jianan Ren, Department of Surgery, Jinling Hospital 305 East Zhongshan Road, Nanjing, 210002, Republic of China, Tel: + 86-2580860108; Fax: +86-25-80860376; E-mail: jiananr@gmail.com

Received August 22, 2014; Accepted November 17, 2014; Published November 19, 2014

Citation: Wang G, Ren J, Hong Z, Wu X, Ren H, et al. (2014) Double "FistulaPatch" Therapy for a Patient with Intestinal and Colonic Enterocutaneous Fistulas. J Trauma Treat 4: 218. doi:10.4172/2167-1222.1000218

Copyright: () 2014 Wang G, et al. This is an open-access article distributed under the terms of the Creative Commons Attribution License, which permits unrestricted use, distribution, and reproduction in any medium, provided the original author and source are credited. 
Citation: Wang G, Ren J, Hong Z, Wu X, Ren H, et al. (2014) Double "Fistula-Patch" Therapy for a Patient with Intestinal and Colonic Enterocutaneous Fistulas. J Trauma Treat 4: 218. doi:10.4172/2167-1222.1000218

Page 2 of 2

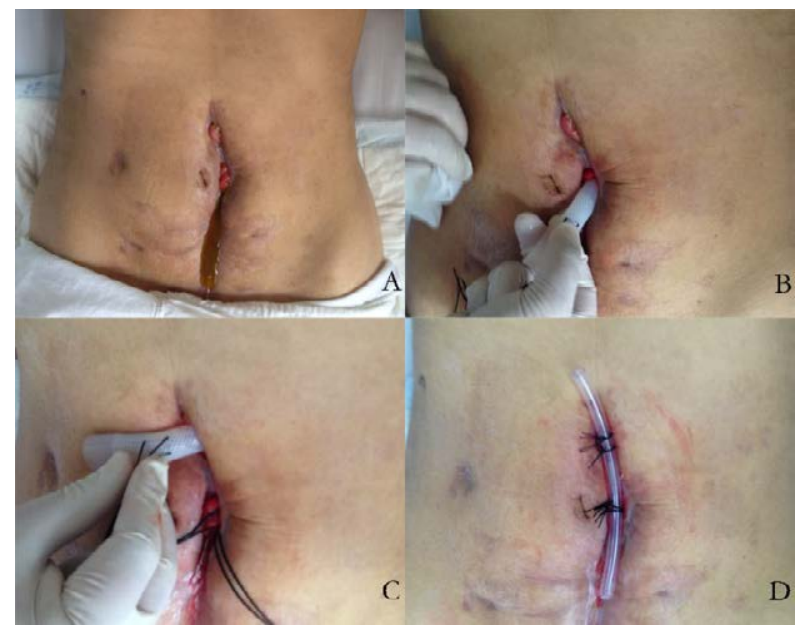

Figure 2: (A) Photograph depicting the patient with two ECFs under abdominal incision. The intestinal ECF (B) and colonic ECF (C) were treated with "fistulapatch" technique. The drainage tube (D) that crossed the ECF was to fix the patch.

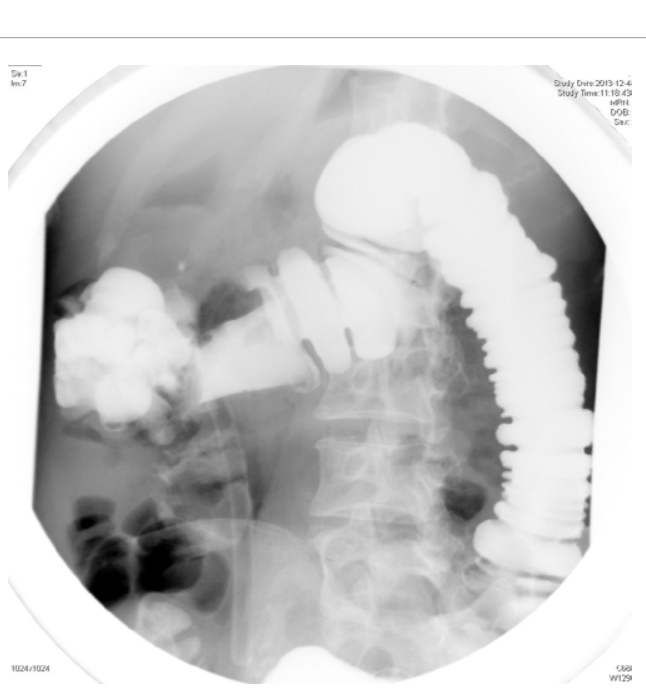

Figure 3: The effect of "fistula-patch" therapy for the colonic ECF was confirmed by barium enema examination without barium leaking.
The definitive surgical management or closure of the fistula is the third and final phase of treatment [7]

When the patient was admitted to our service, she was in the second phase, and the key point of treatment was how to supply enteral nutrition and wound care. We had reported our work which named as "fistula-patch" technique for controlling contamination of intestinal fistula draining into the open abdominal wounds, and applying enteral nutrition in EAF patients. This technique is simple but works. So we designed double "fistula-patch" for two ECFs of the patient. The successful restore of intestinal continuity by the patch was confirmed by barium enema examination and CT examination.

The "fistula-patch" technique could be conducted once peritoneal sepsis has been controlled and small-bowel function has resumed (indicated by reduced nasogastric aspirate and active stoma output). The technique is a simple method to control ECF, with the benefits of avoiding loss of intestinal effluents, simplifying wound care, and applying enteral nutritions. What is more, the patch would remain in situ until definite operations for fistulas. This innovative strategy provides an easier and promising option in the management of ECF, or even multiple ECFs.

\section{Acknowledgment}

This work was supported by Grants from the medical science project of Nanjing military region of China (MS111) and Chinese army medical science research project (CNJ13C007)

\section{References}

1. Lloyd DA, Gabe SM, Windsor AC (2006) Nutrition and management of enterocutaneous fistula. Br J Surg 93: 1045-1055.

2. Schecter WP (2011) Management of enterocutaneous fistulas. Surg Clin North Am 91: 481-491.

3. Polk TM, Schwab CW (2012) Metabolic and nutritional support of the enterocutaneous fistula patient: a three-phase approach. World J Surg 36: 524-533.

4. Ham M, Horton K, Kaunitz J (2007) Fistuloclysis: case report and literature review. Nutr Clin Pract 22: 553-557.

5. Wu Y, Ren J, Wang G, Zhou B, Ding C, et al. (2014) Fistuloclysis improves liver function and nutritional status in patients with high-output upper enteric fistula. Gastroenterol Res Pract 2014: 941514.

6. Wang G, Ren J, Liu S, Wu X, Gu G, et al. (2013) "Fistula patch": making the treatment of enteroatmospheric fistulae in the open abdomen easier. J Trauma Acute Care Surg 74: 1175-1177.

7. Bleier JI, Hedrick T (2010) Metabolic support of the enterocutaneous fistula patient. Clin Colon Rectal Surg 23: 142-148. 